Check for updates

Cite this: DOI: 10.1039/c7sm00345e

\title{
Cooperative wrapping of nanoparticles of various sizes and shapes by lipid membranes $\dagger$
}

\author{
Kai Xiong, ${ }^{a}$ Jiayin Zhao, ${ }^{b}$ Daowen Yang, ${ }^{c}$ Qingwen Cheng, ${ }^{d}$ Jiuling Wang (D) *ef and \\ Hongbing Ji*g
}

\begin{abstract}
Understanding the interaction between nanoparticles (NPs) and cell membranes is crucial for the design of NP-based drug delivery systems and for the assessment of the risks exerted by the NPs. Recent experimental and theoretical studies have shown that cell membranes can mediate attraction between NPs and form tubular structures to wrap multiple NPs. However, the cooperative wrapping process is still not well understood, and the shape effect of NPs is not considered. In this article, we use largescale coarse-grained molecular dynamics (CGMD) simulations to study the cooperative wrapping of NPs when a varying number of NPs adhered to the membrane. Spherical, prolate and oblate NPs of different sizes are considered in this study. We find that, in addition to tubular structures, the membrane can form a pocket-like and a handle-like structure to wrap multiple NPs depending on the size and shape of the NPs. Furthermore, we find that NPs can mediate membrane hemifusion or fusion during this process. Our findings provide new insights into the interaction of NPs with the cell membrane.
\end{abstract}

Received 19th February 2017 Accepted 16th June 2017

DOI: $10.1039 / \mathrm{c} 7 \mathrm{sm} 00345 \mathrm{e}$

rsc.li/soft-matter-journal between spherical NPs, ${ }^{39,40}$ and drive the linear aggregation of adsorbed NPs on the lipid membrane, ${ }^{41,42}$ which further initiates the formation of a membrane tube to wrap multiple NPs when increasing the binding constant between the NPs and the cell membrane. ${ }^{43,44}$ Theoretical analysis shows that the energy gain for cooperative wrapping of NPs in membrane tubes is larger than individual wrapping of NPs. ${ }^{45,46}$

However, cooperative wrapping of NPs is still far from being fully understood. ${ }^{43-49}$ The present theoretical analysis only obtains the system energy of different wrapping phases and lacks the dynamic process of cooperative wrapping. ${ }^{50}$ In particular, it cannot capture the membrane structure transition when the number of NPs gradually increases. In addition, there are few studies focused on the cooperative wrapping of NPs of various shapes. For prolate NPs, theoretical analysis indicates that cooperative wrapping in membrane tubes is highly stable compared to individual wrapping. ${ }^{46}$ However, the tubular structure needs to wrap at least one strongly curved tip of the prolate NPs, which is highly unfavorable from the viewpoint of energy. This barrier may prevent the formation of the tubular structure. In fact, experimental and theoretical studies have shown that local geometrical properties such as the local mean curvature of NPs would significantly affect the wrapping behavior of lipid membranes on NP surfaces. ${ }^{11,25,51}$ For example, the local mean curvature of NPs at the point of initial contact with macrophages determines whether the cells internalize or simply spread on NPs. ${ }^{11}$ For oblate NPs, Weikl et al. predicted that the membrane forms a tubular structure, in which the more strongly curved edges of the neighboring NPs face each other, to wrap 
multiple NPs. ${ }^{45}$ Such a tubular structure does not exhibit rotational symmetry, thereby it has not been studied before.

In this article, we carry out large-scale coarse-grained molecular dynamics (CGMD) simulations to investigate cooperative wrapping of multiple NPs of various shapes by lipid membranes. Molecular dynamics (MD) simulations have successfully reproduced the particle size effect, shape effect and stiffness effect during the cellular uptake of NPs. ${ }^{10,19,30,52-55}$ The orientation changes of NPs during the internalization process have also been captured by MD simulations. ${ }^{21,23,24}$ Here, we adopt the solventfree coarse-grained lipid membrane model developed by Deserno et al., which allows us to explore a lipid membrane system with length scales of hundreds of nanometers and timescales of milliseconds. ${ }^{56,57}$ We investigate cooperative wrapping when a varying number of NPs adhered to the membrane, and focus on the size and shape (spherical, prolate, and oblate NPs) effects during this process.

\section{Model and methods}

We adopted the solvent-free lipid membrane model developed by Deserno et al. to model a large piece of lipid membrane efficiently, with which lipids can assemble into a fluid membrane without water. ${ }^{56,57}$ Using this model, we can simulate a lipid membrane with hundreds of nanometers, which is beyond the capacity of explicit solvent lipid models now. ${ }^{48,50}$ Each lipid molecule is represented by one hydrophilic head bead and two hydrophobic tail beads, as shown in Fig. 1a. In the snapshots, the hydrophilic head and hydrophobic tails are shown in red and cyan. Such a model has been successfully applied to investigate the aggregation and vesiculation of membrane proteins adsorbed on the membrane. ${ }^{39}$ The interaction between the different beads is described in the ESI. $\dagger$

The initial box size was $150 \sigma \times 150 \sigma \times 150 \sigma(\sigma$ is the unit of length), and a periodic boundary condition was applied in the simulation. The membrane was composed of 40504 lipids and was located in the $x y$-plane. We used a Nose-Hoover barostat to control the system pressure at $0.001 \varepsilon / \sigma^{3}$ ( $\varepsilon$ is the unit of energy) in the $x$-and $y$-dimensions with a stress damping parameter of

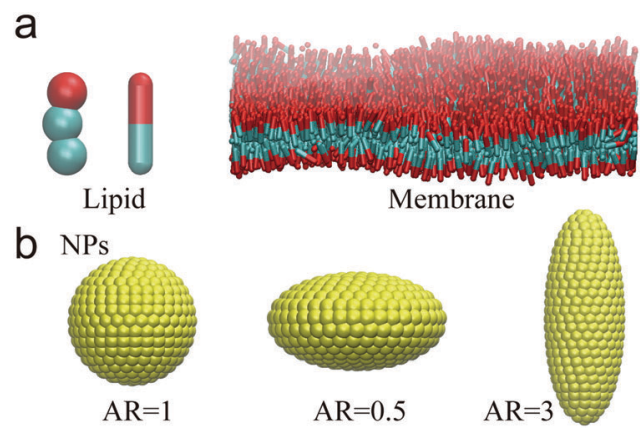

Fig. 1 Illustration of the individual components used in our simulation. (a) Representation of a lipid by the drawing method of beads and licorice, and a patch of membrane. (b) NPs with different AR values; the $D$ or $D_{v}$ of these NPs is $8 \sigma$. Lipid heads are shown in red and the lipid tail in cyan, and NPs are shown in yellow.
$10 \tau$ ( $\tau$ is the unit of time), and the pressure components in the $x$ - and $y$-dimensions were coupled, while the height of the box (z-dimension) was kept constant. ${ }^{39,51,58}$ Through this method, we obtained a lipid membrane with a constant membrane tension, which is about $1.9 \times 10^{-2} \mathrm{pN} \mathrm{nm}^{-1}$ (ESI $\left.\dagger\right)$. We utilized the velocity-Verlet algorithm to perform time integration, and a Langevin thermostat to control the system temperature at $k_{\mathrm{B}} \mathrm{T}=1.1 \varepsilon$ with a friction constant $\Gamma=\tau^{-1}$, where $k_{\mathrm{B}}$ is the Boltzmann constant and $T$ is the temperature. The integration time step was $\delta t=0.01 \tau$, where $\tau=\sqrt{m \sigma^{2} / \varepsilon}$ is the units of time with $m$ the bead mass, and $\tau$ is about 10 ns. All simulations were implemented with the LAMMPS package. $x^{59}$

The shape of NPs is defined by the equation $\frac{x^{2}+y^{2}}{a^{2}}+\frac{z^{2}}{b^{2}}=1$ in the Cartesian coordinates $x, y$, and $z$, where $a$ and $b$ are the semi-axes of the ellipsoid in the $x y$-plane and in the $z$-direction. The aspect ratio (AR) of NPs is defined as $\mathrm{AR}=b / a$, where $\mathrm{AR}=1$ for spherical NPs, AR $>1$ for prolate NPs, and AR $<1$ for oblate NPs. The diameter of spherical NPs is denoted by $D$. The equivalent diameter $D_{\mathrm{v}}$ of ellipsoidal NPs is defined as the diameter of the sphere having the same volume as the ellipsoid, and $D_{\mathrm{v}}$ can be expressed as $D_{\mathrm{v}}=2 \sqrt[3]{a^{2} b}$. We constructed a group of NPs with an AR from 0.5 to 3 and $D$ or $D_{\mathrm{v}}$ from $6 \sigma$ to $10 \sigma$ (Fig. 1b and Fig. S1, ESI $\dagger$ ). The surface bead density of all the NPs is $1.767 / \sigma$, and the mass of all the beads is $1.0 \mathrm{~m}$. The interaction between NPs and lipid membranes is described by the LennardJones (LJ) potential: $U_{\mathrm{LJ}}(r)=4 \alpha \varepsilon\left[\left(\frac{b}{r}\right)^{12}-\left(\frac{b}{r}\right)^{6}\right]$, where $\alpha$ is the interaction strength and is set as $\alpha=1$. The individual NP is constrained as a rigid body during the simulation.

At the beginning of each simulation, one NP is placed $2 \sigma$ above the equilibrated lipid membrane. Typically, a simulation time of $240000 \tau$ is sufficient to obtain a stable NP-membrane structure. For some cases (for example, oblate NPs with $D_{\mathrm{v}}$ values of $8 \sigma$ and $6 \sigma$ ), if the system configuration changes significantly in the second half of the simulation, we will extend the simulation time accordingly. After obtaining a stable system configuration, we add another NP to the system, not far from the previously formed membrane-NP structure. Using this method, we can add NPs to the system continuously and obtain the membrane structure wrapping different numbers of NPs. We add up to 10 NPs to the system and observe the dynamics of their interactions. In addition, we also compare these results with the cases when we add multiple NPs to the system simultaneously.

In this article, we obtain the wrapping fraction of NPs by the lipid membrane. The wrapping fraction is defined as follows: the total number of beads in one NP is $N_{\mathrm{t}}$, the number of beads that are within $1.3 \sigma$ of any lipid is $N_{\mathrm{w}}$, and the wrapping fraction $f$ is $f=N_{\mathrm{w}} / N_{\mathrm{t}}$.

\section{Results and discussion}

\subsection{Wrapping of multiple spherical NPs}

Spherical NPs are the first generation of NPs used as drug delivery vectors. In this article, we first study the interaction between spherical NPs of different sizes and lipid membranes. 
We fixed the interaction strength at $\alpha=1$, and changed the diameter of the spheres from $6 \sigma$ to $10 \sigma$. To illustrate the implication of this adhesion strength, we obtained the wrapping fractions of individual spherical NPs as a function of the adhesion strength (Fig. S2, ESI $\dagger$ ). The general trend is that the wrapping fraction increases with increasing adhesion strength. Meanwhile, the wrapping fraction increases slowly with the adhesion strength $\alpha$ when $\alpha$ is small, then the wrapping fraction increases significantly for intermediate $\alpha$ values, and finally the wrapping fraction increases slowly again for large $\alpha$ values until the NPs are completely wrapped by the lipid membrane. These results are quite consistent with the theoretical predictions by Weikl et al., in which the effect of the potential range of the NP-membrane interaction is taken into account. ${ }^{45}$ With the adhesion strength fixed at $\alpha=1$, the wrapping processes of the NPs are displayed in Fig. 2, and the interaction energy profiles are shown in Fig. S3 (ESI $\dagger$ ). It is shown that the membrane would form different membrane structures to wrap multiple NPs, which is governed by the interplay of bending and adhesion energy.

As shown in Fig. 2a, spherical NPs with a diameter of $10 \sigma$ can be internalized by the lipid membrane rapidly and detached from the membrane. The kinetics of endocytosis is similar to that of recent molecular simulation studies. ${ }^{10,14,51}$ When the diameter of the spherical NPs is reduced to $8 \sigma$, individual NPs cannot be completely wrapped by the lipid membrane, and the wrapping fraction of the NPs is about 0.85 (Fig. $2 \mathrm{~b}$ and $3 \mathrm{a}$ ). These results are consistent with the size effect of the cellular uptake of NPs found in recent experimental and theoretical studies. ${ }^{6-10}$ Partial wrapping is due to the long-range attraction potential adopted here to describe the interaction between NPs and the lipid membrane, whose range $(2.5 \sigma)$ is comparable to the size of the NPs. And the partial wrapping state occurs from a subtle interplay of bending and adhesion energies in the region where the membrane detaches from the NPs. ${ }^{45}$ In order to investigate cooperative wrapping of NPs, we added another NP to the simulation system, as shown in Fig. 2b. We find that the two NPs are gathered together and wrapped by a tubular membrane. The wrapping fraction of the second NP is 0.77 , while that of the first NP is slightly increased to 0.9 (Fig. 3a). We observe the same tubular structure when more NPs are added to the system. This phenomenon is consistent with the recent theoretical and Monte Carlo simulation results that the lipid
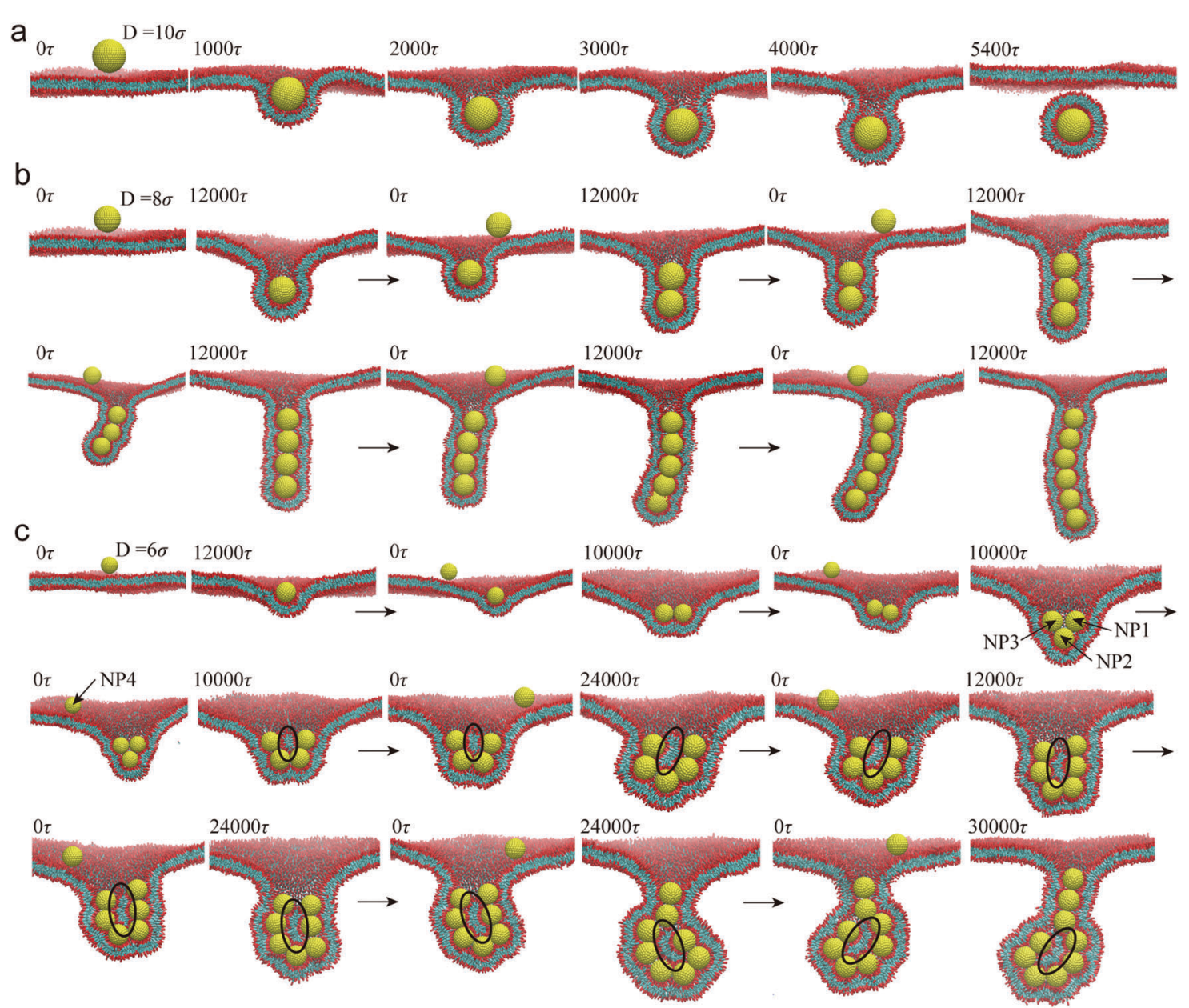

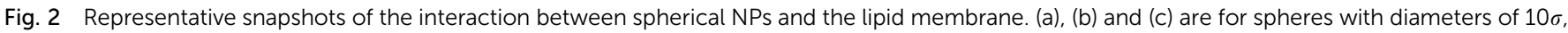

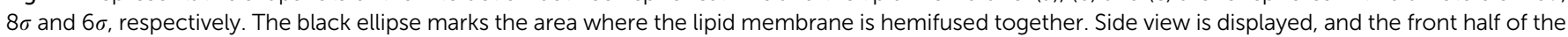
lipid membrane is not shown for clarity. 
a

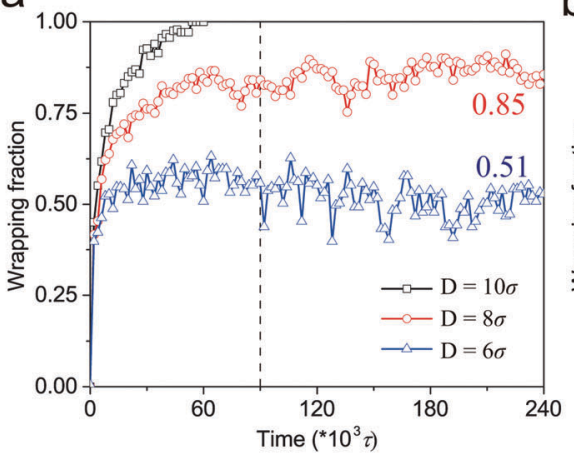

b

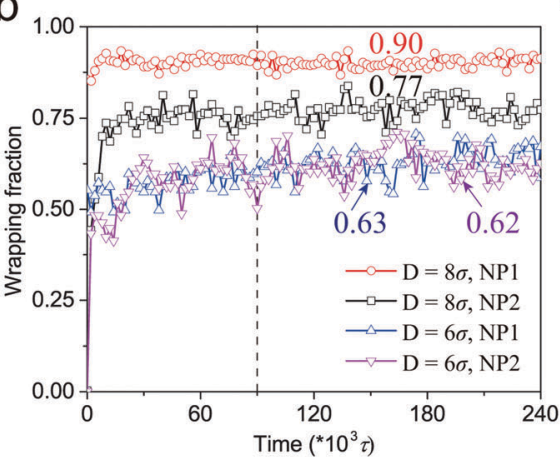

C

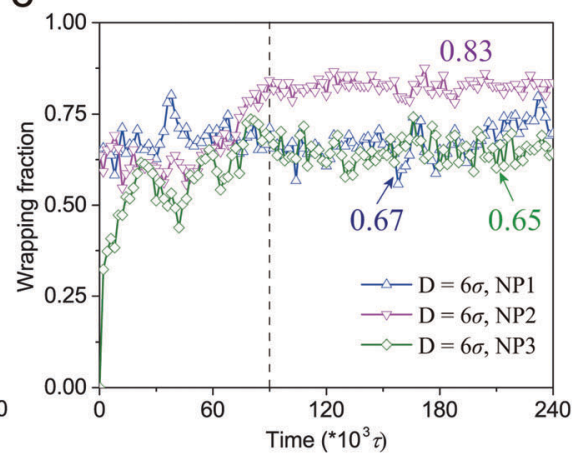

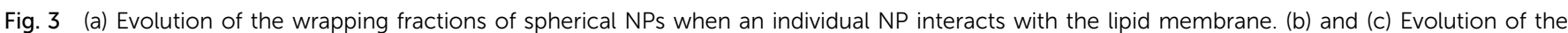

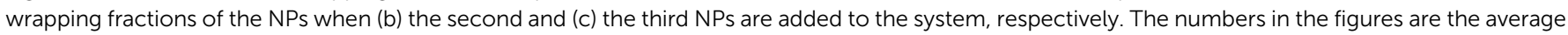
wrapping fractions of the NPs from the time indicated by the dotted line to the end of the simulations.

membrane can mediate the attraction between spherical NPs and form a tubular structure to wrap multiple NPs. ${ }^{39,40,43-45}$

Next we continue to reduce the diameter of the spherical NPs to $6 \sigma$. The wrapping fraction of an individual NP (NP1) is reduced to 0.51 (Fig. 2c and $3 \mathrm{a}$ ), and the fraction increases to 0.63 when the second NP (NP2) is added to the system (Fig. 2c and $3 \mathrm{~b})$. The increased wrapping fraction of the NPs clearly demonstrates the cooperative effect in cellular uptake. When the third NP (NP3) is added to the system, the three NPs are brought into close contact with each other, and the distance between them is about $7 \sigma$ (Fig. 4a). Moreover, the wrapping fraction of NP2 increases to 0.83 (Fig. 2c and 3c), an increase of $63 \%$ compared to the case of individual wrapping. When the fourth NP (NP4) is added, it will approach NP3, meanwhile NP3 will leave NP2 (Fig. 4b), and finally the lipid membrane will form a pocket-like structure to wrap these four NPs (Fig. 2c). Furthermore, the lipid membranes on both sides of the NPs are hemifused together to increase the wrapping fraction of the NPs (Fig. S4, ESI $\dagger$ ); the hemifusion area is marked by a black ellipse in Fig. $2 \mathrm{c}$ and is analyzed in the ESI, $\dagger$ Fig. S5. Hemifusion occurs because the adhesion between the NPs and the membrane leads to the spread of the membrane on the surface of the NPs, which would reduce the distance between the membrane patches, thereby facilitating membrane hemifusion. Meanwhile, the local membrane tension and high curvature of the membrane in the contact front may promote membrane hemifusion during this process. ${ }^{60,61}$ NP-mediated membrane fusion is a novel discovery, ${ }^{62,63}$ and it is worth using all-atom MD simulations and carefully designed experiments to confirm our findings. As more NPs are added to the system, the area of membrane hemifusion increases, and the pocket-like structure grows. Unlike the spherical bud model proposed by Wang et $a l .{ }^{47}$ the pocket-like structure does not have rotational symmetry, and all the NPs are distributed in the same plane. The pocket-like structure (or loop structure) has also been observed transiently in Monte Carlo simulations as the organization of the NPs changed from linear aggregation to tubular protrusion. ${ }^{44}$ The energy barrier during this transition process is easily overcome in the Monte Carlo simulation, because the overall system adhesion energy gain can compensate for the local bending energy cost. However, in our MD simulations, the molecular details of the membrane have been considered. For NPs with a diameter close to the membrane thickness, the bending energy would increase drastically when the NPs are
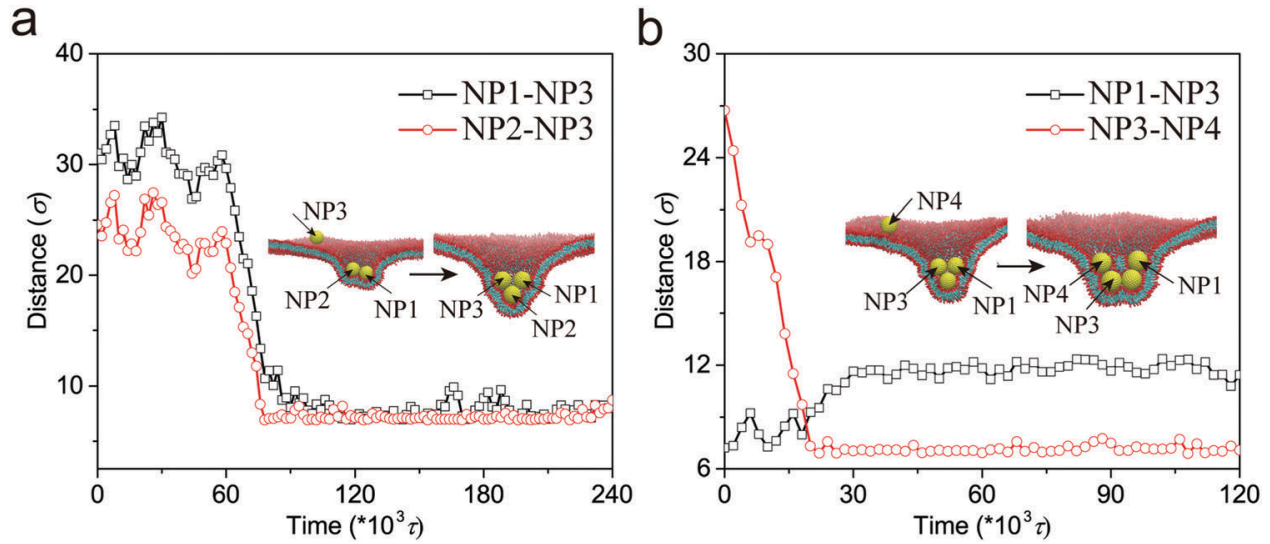

Fig. 4 Evolution of the distance between NPs when (a) the third and (b) the fourth NPs with a diameter of $6 \sigma$ are added to the system. The distance in this paper is defined as the distance between the centroids of the NPs. 
wrapped in depth. The interplay of bending and adhesion energies causes the NPs to be wrapped only to a certain extent, thus such a structure is kinetically trapped in the simulation. When the eighth NP is added to the system, the pocket is tightened and the uppermost two NPs will be wrapped by a tubular membrane structure (Fig. 2c). The tubular structure will continue to grow as more NPs are added. In general, the size effect in wrapping of multiple NPs by the lipid membrane is obvious, which has also been observed in the Monte Carlo simulations. ${ }^{44}$ Due to the consideration of the molecular details here, we also captured the occurrence of membrane hemifusion.

We also study the case when multiple NPs are added to the system simultaneously for comparison. For spherical NPs with a diameter of $10 \sigma$, depending on the separation of the NPs, the two NPs may be cooperatively or individually wrapped by the lipid membrane (Fig. S6, ESI $\dagger$ ). For small spherical NPS
$(D=8 \sigma)$, the membrane forms tubular structures to wrap multiple NPs, and sometimes several NPs can be completely wrapped by the membrane tube and detached from the membrane (Fig. S7a, ESI $\dagger$ ). For smaller spherical NPs $(D=6 \sigma)$, when four NPs are added simultaneously, the membrane forms a pocket-like structure to wrap these NPs, similar to the case when the NPs are added one by one (Fig. S7d, ESI $\dagger$ ). When six or nine NPs are added simultaneously, the membrane forms a handle-like structure to wrap these NPs, in contrast to the pocket-like structure when the NPs are added one by one (Fig. S7e and f, ESI $\dagger$ ).

\subsection{Wrapping of multiple prolate NPs}

Usually, prolate NPs are more difficult than spherical NPs to be fully wrapped. ${ }^{17}$ Here we first consider three prolate NPs with an AR of 3 but of different sizes.
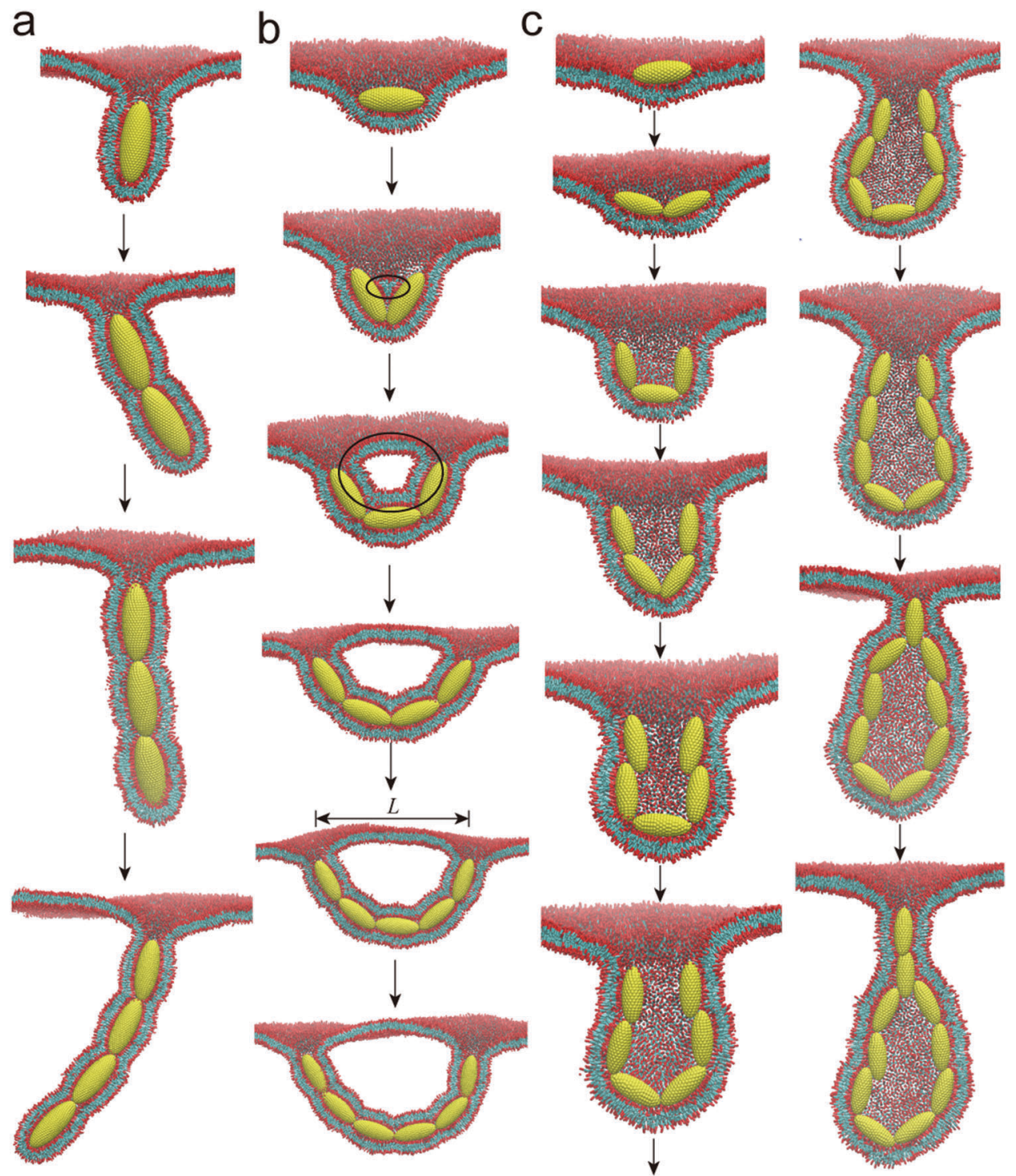

Fig. 5 Representative snapshots of the interaction between prolate NPs (AR $=3$ ) and the lipid membrane. (a) is for NPs with a $D_{\vee}$ of $10 \sigma$; (b) and (c) are for NPs with $D_{\vee}$ values of $8 \sigma$ and $6 \sigma$. Side view is displayed and the front half of the lipid membrane is not shown for clarity. $L$ in (b) denotes the length of the handle-like structure. 
A single prolate NP with a $D_{\mathrm{v}}$ of $10 \sigma$ cannot be completely wrapped by the lipid membrane, one tip of which is uncoated (Fig. 5a and Fig. S8, ESI $\dagger$ ). In fact, the mean curvature at the tips of the prolate NPs is quite large and the corresponding energy consumption by membrane wrapping is relatively high, which impedes the attachment of the membrane and the subsequent fission of the membrane neck. ${ }^{64}$ When another NP is added to the system, the lipid membrane forms a tubular structure to wrap the two NPs, which agrees well with previous theoretical predictions. ${ }^{43-46}$ Adding more NPs to the system does not change the cooperative wrapping manner, but only increases the length of the tubular structure (Fig. 5a). The wrapping fraction of the NPs is about 0.96 (Fig. 6a and Fig. S9, ESI $\dagger$ ).

For a small single prolate NP $\left(D_{\mathrm{v}}=8 \sigma\right)$, it lies flat on the surface of the membrane with a wrapping fraction of 0.65 , and the major axis of the NP is parallel to the membrane (Fig. 5b). The NP cannot rotate itself and be wrapped further because the adhesion energy is insufficient to compensate for the bending energy cost at the tip of the NP. ${ }^{22,23,25}$ When the second NP is added to the system, the two NPs gather together and the wrapping fraction of NPs increases to 0.89 (Fig. $5 \mathrm{~b}$ and 6b). Meanwhile, the lipid membrane is hemifused, and the hemifusion area is marked by a black ellipse in Fig. 5b. When the third NP is added, the membrane is fused together and the fusion area is separated from the membrane, and the lipid membrane forms a handle-like structure (a curved membrane tube, both ends of which are connected to the membrane) to wrap the three NPs. The wrapping fraction of the NPs is even increased to 0.93 (Fig. S10, ESI $\dagger$ ). As more NPs are added to the system, the length of the handle increases and the cooperative wrapping manner does not change (Fig. S11, ESI $\dagger$ ).

For a smaller single prolate NP $\left(D_{\mathrm{v}}=6 \sigma\right)$, it adheres to the membrane surface with a wrapping fraction of 0.53 (Fig. $5 \mathrm{c}$ and 6a). When the second NP is added to the system, the two NPs linearly aggregate together and the wrapping fraction of the NPs increases to 0.73 (Fig. 5c and 6c). In fact, for these very small prolate NPs, wrapping the strongly curved tips is highly energetically unfavorable (Fig. S12, ESI $\dagger$ ). When the third NP is added, the lipid membrane forms a pocket-like structure to wrap the three NPs, the wrapping fraction of which is further increased to 0.86 and 0.89 (Fig. S13, ESI $\dagger$ ). The presence of multiple NPs significantly increases the wrapping fraction of the NPs, which again confirms the cooperative effect in wrapping of the NPs by the lipid membrane. In addition, the lipid membrane is not fused during this process. As more NPs are added, the pocket becomes deeper to accommodate more NPs (Fig. 5c). When the ninth NP is added, the pocket is tightened and the NP is in the seal. When the tenth NP is added, the uppermost two NPs will be wrapped in a tubular membrane, which is similar to the case of spherical NPs with a diameter of $6 \sigma$, except that the inside of the pocket is hollow. As more NPs are added, we speculate that the tubular membrane portion will grow and the NP will fill inside it.

We also investigated the interaction between prolate NPs with an AR of 2 with the lipid membrane (Fig. S14 and S15, ESI $\dagger$ ). For NPs with $D_{\mathrm{v}}$ values of $10 \sigma$ and $8 \sigma$, the cooperative wrapping manner of the NPs is similar to that of the NPs with an AR of 3. For NPs with a $D_{\mathrm{v}}$ of $6 \sigma$, the lipid membrane is hemifused when they form a pocket-like structure to wrap multiple NPs. Therefore, depending on the size of the NPs, the lipid membrane will form a tubular structure, a handle-like structure or a pocket-like structure to wrap them. We also study the case when multiple prolate NPs $(\mathrm{AR}=3)$ are added to the system simultaneously (Fig. S16, ESI $\dagger$ ). The results show that the membrane forms pocket-like structures to wrap multiple NPs with $D_{\mathrm{v}}$ values of $8 \sigma$ and $6 \sigma$. Thus, the order in which the NPs are added may affect the final NP-membrane complex structure; however, the cooperative effect is common for these NPs.

\subsection{Wrapping of multiple oblate NPs}

Similar to prolate NPs, we first consider three oblate NPs with an AR of 0.5 but of different sizes. Single oblate NPs with a $D_{\mathrm{v}}$ of $10 \sigma$ can be fully wrapped by the membrane (Fig. 7a, 8a and Fig. S17a, ESI $\dagger$ ). The internalization process is accompanied by orientation changes of the NPs, which is consistent with the previous theoretical prediction and numerical simulations. ${ }^{22,51}$ For a small single oblate NP $\left(D_{\mathrm{v}}=8 \sigma\right)$, it lies flat on the a

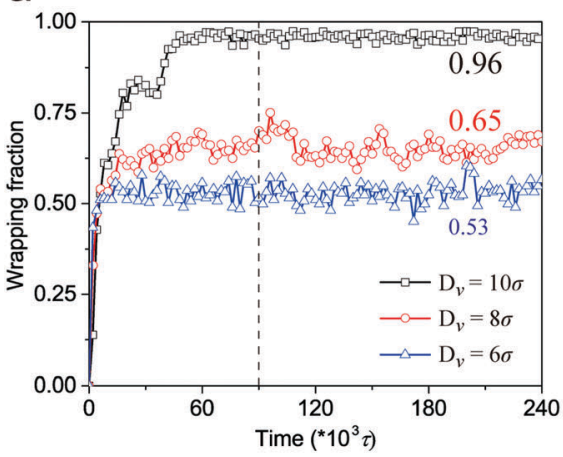

b

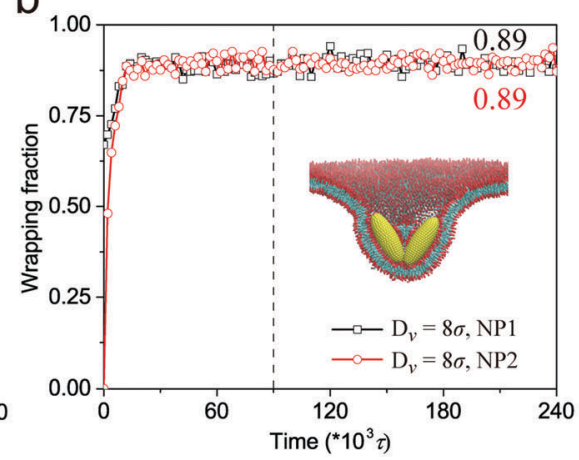

C

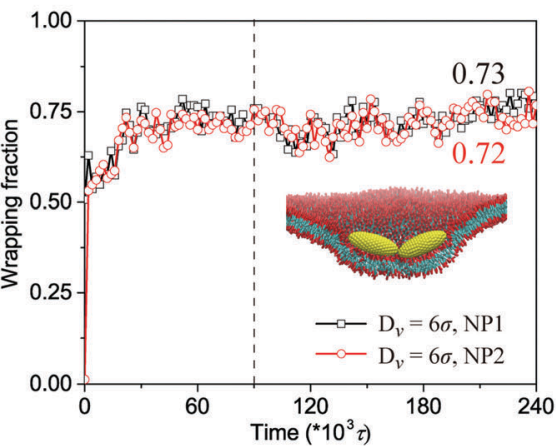

Fig. 6 (a) Evolution of the wrapping fractions of prolate NPs $(A R=3)$ when an individual NP interacts with the lipid membrane. (b) and (c) Evolution of the wrapping fractions of prolate NPs when the second NP is added to the system, (b) is for NPs with AR $=3$ and $D_{v}=8 \sigma$, and (c) is for NPs with AR $=3$ and $D_{\mathrm{v}}=6 \sigma$. The numbers in the figures are the average wrapping fractions of the NPs. 


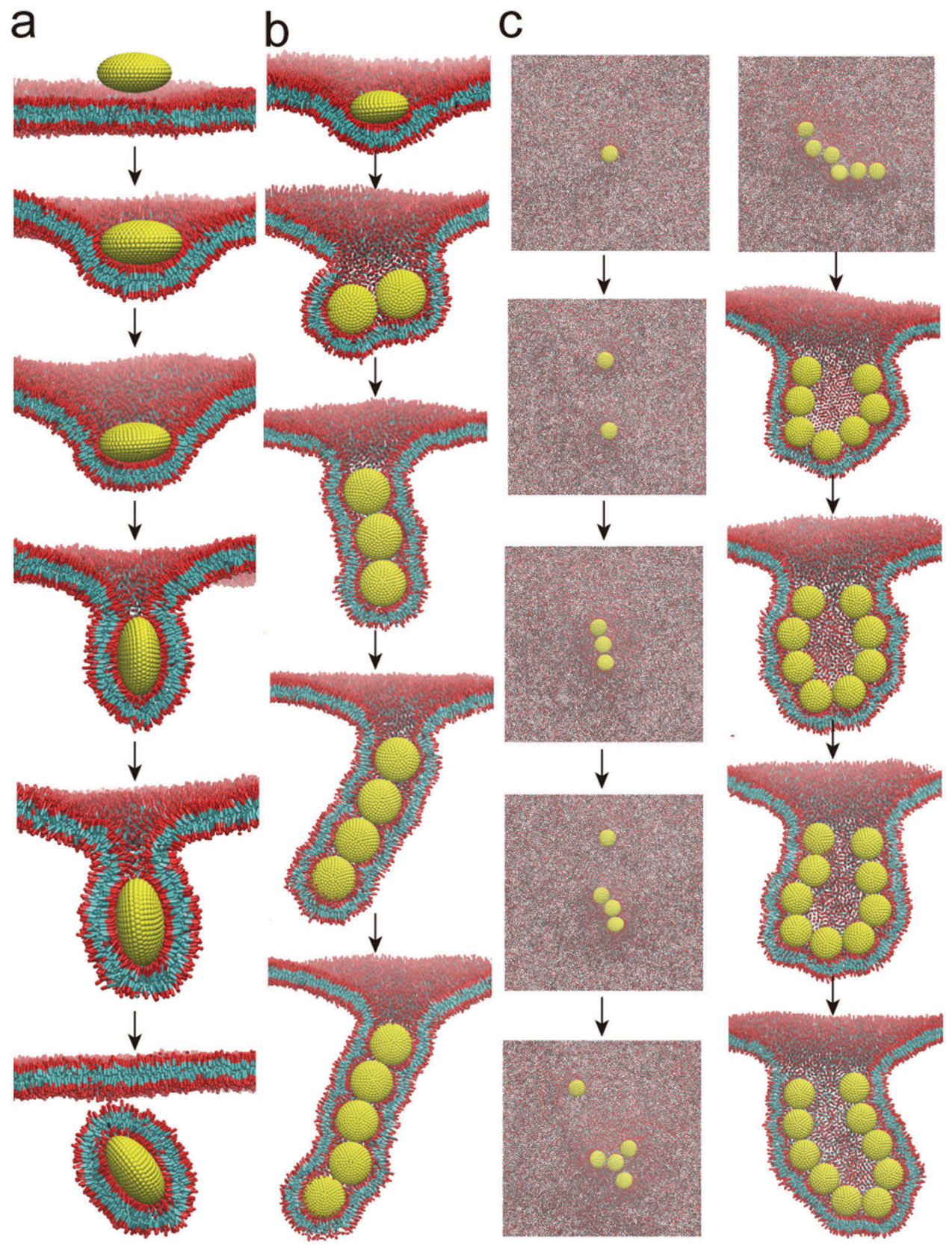

Fig. 7 Representative snapshots of the interaction between oblate NPs (AR $=0.5)$ and the lipid membrane. (a) is for NPs with a $D_{v}$ of $10 \sigma$; (b) and (c) are for NPs with $D_{v}$ values of $8 \sigma$ and $6 \sigma$. The top six figures in (c) are top views, other figures are side views, and the front half of the lipid membrane is not shown for clarity.

membrane and the wrapping fraction of the NP is 0.55 (Fig. $7 \mathrm{~b}$ and $8 \mathrm{a}$ ). When the second NP is added to the system, they gather together, rotate, and the curved edges face each other. The wrapping fraction of the NPs increases to 0.89 (Fig. 8b). When more NPs are added, the lipid membrane forms a tubular structure to wrap these NPs, and the wrapping fraction can even reach 0.94 (Fig. S18, ESI $\dagger$ ).

For a smaller oblate NP $\left(D_{\mathrm{v}}=6 \sigma\right)$, the wrapping fraction of an individual NP is 0.52 (Fig. 7c and 8a). When the second NP is added to the system, the two NPs approach each other several times and eventually move away from each other (Fig. S19, ESI†).
The wrapping fraction of the NPs is still 0.52 (Fig. S20a, ESI $\dagger$ ). When the third NP is added, they will linearly gather together, and the wrapping fraction of the NPs does not change (Fig. S20b and S21, ESI + ). However, after the addition of the fourth NP, we do not find it to be clustered with the other three NPs during our simulation time (Fig. S22, ESI $\dagger$ ). When the fifth NP is added, we even find that four of the NPs are isotropically clustered together (Fig. S23, ESI $\dagger$ ). We speculate that the attraction of oblate NPs mediated by the lipid membrane is weaker than that of spherical NPs, which causes the NP to leave the cluster under thermal fluctuation. When the sixth NP is added, all NPs linearly 
a

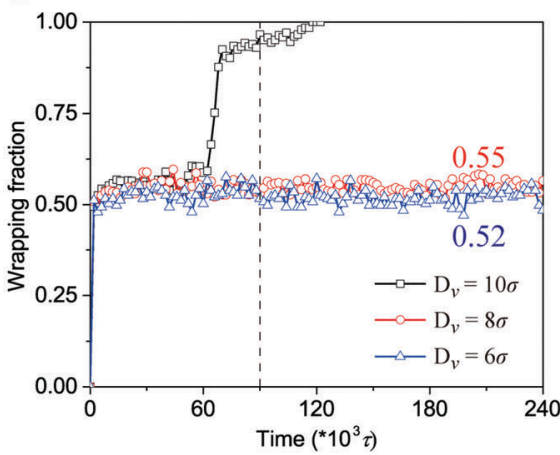

b

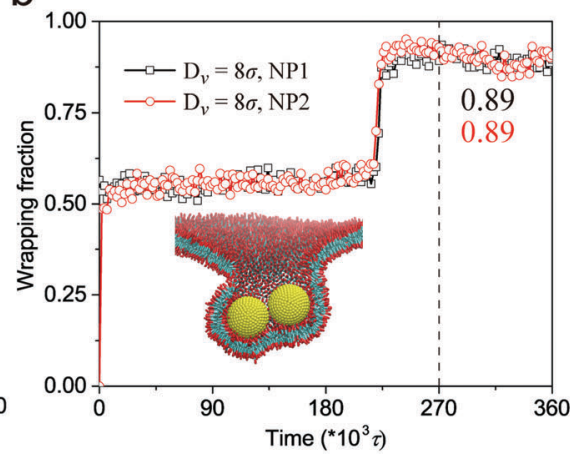

C

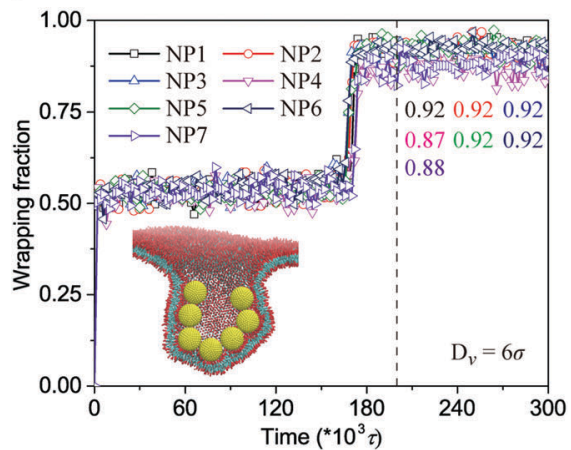

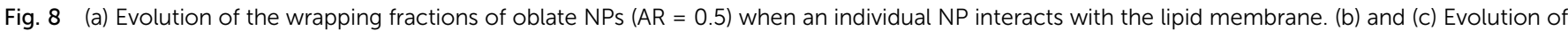

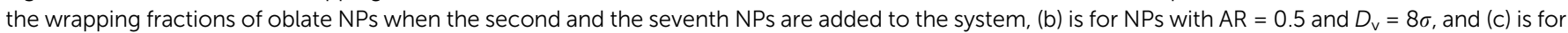
NPs with AR $=0.5$ and $D_{v}=6 \sigma$. The numbers in (a), (b) and (c) are the average wrapping fractions of the NPs.

aggregate together again (Fig. S24, ESI $\dagger$ ). So far, the wrapping fraction of the NPs has remained at 0.52 (Fig. S20, ESI $\dagger$ ). When the seventh NP is added, all NPs first linearly gather together, then all NPs rotate almost simultaneously from lying flat on the membrane surface to an erect position, and finally the membrane forms a pocket-like structure to wrap all these particles (Fig. 7c and Video S1, ESI $\dagger$ ). Meanwhile, the interaction energy between the NPs and the membrane decreases drastically (Fig. S17, ESI $\dagger$ ), and the wrapping fraction of the NPs increases to 0.92 (Fig. 8c). As more NPs are added, the pocket becomes deeper (Fig. 7c). Note that we have added up to 15 NPs to the system, and we did not find pocket tightening during our simulation.

We also consider oblate NPs with an AR of 0.75 (Fig. S25 and S26, ESI $\dagger$ ). For NPs with a $D_{\mathrm{v}}$ of $10 \sigma$, they can also be fully wrapped by the lipid membrane. For NPs with a $D_{\mathrm{v}}$ of $8 \sigma$, the membrane forms a tubular structure to wrap multiple NPs. For NPs with a $D_{\mathrm{v}}$ of $6 \sigma$, when the number of NPs is not more than 7 , the lipid membrane forms a pocket-like structure to wrap the NPs; when the number of NPs exceeds 7, the lipid membrane forms a tubular structure over the pocket structure to wrap these NPs (Fig. S25, ESI $\dagger$ ). When multiple oblate NPs $(\mathrm{AR}=0.5)$ are added to the system simultaneously (Fig. S27, $\mathrm{ESI} \dagger)$, we found that the membrane prefers to form pocket-like structures to wrap multiple NPs with $D_{\mathrm{v}}$ values of $8 \sigma$ and $6 \sigma$.

\section{Conclusions}

In summary, through large-scale CGMD simulations, we have studied the cooperative wrapping of NPs with different sizes and shapes by lipid membranes. Our simulations vividly show the cooperative wrapping process of NPs, and clearly show that the wrapping fraction of the NPs increases significantly when multiple NPs are present in the system. For spherical NPs, large individual NPs $(D=10 \sigma)$ can be completely wrapped by the lipid membrane, while several small NPs $(D=8 \sigma)$ will be wrapped in a tubular membrane structure, and multiple smaller NPs $(D=6 \sigma)$ are wrapped in a pocket-like membrane structure.
For prolate NPs, a single large NP $\left(D_{\mathrm{v}}=10 \sigma\right)$ cannot be completely wrapped by the lipid membrane, one tip of which is not wrapped due to the large curvature. A group of small NPs $\left(D_{\mathrm{v}}=8 \sigma\right)$ will be wrapped in a handle-like membrane structure, and smaller NPs $\left(D_{\mathrm{v}}=6 \sigma\right)$ are wrapped in a pocket-like membrane structure. For oblate NPs, a single large NP $\left(D_{\mathrm{v}}=10 \sigma\right)$ can be completely wrapped by the lipid membrane, and a group of small NPs $\left(D_{\mathrm{v}}=8 \sigma\right)$ will be wrapped in a tubular membrane structure, while smaller NPs $\left(D_{\mathrm{v}}=6 \sigma\right)$ are wrapped in a pocket-like membrane structure. At the same time, we find that NP-mediated membrane hemifusion or fusion occurs for different shapes of NPs during the simulation, which is worth further study. Our results can be used to design NPs for drug delivery in nanomedicine, as well as to understand the toxicity mechanism of NPs to environment and human beings.

\section{Acknowledgements}

We are grateful for the financial support from the National Natural Science Foundation of China (No. 41173113 and 41473122) and the Hundred Talents Program of Chinese Academy of Sciences.

\section{References}

1 R. A. Petros and J. M. DeSimone, Nat. Rev. Drug Discovery, 2010, 9, 615-627.

2 I. Canton and G. Battaglia, Chem. Soc. Rev., 2012, 41, 2718-2739.

3 A. Nel, T. Xia, L. Madler and N. Li, Science, 2006, 311, 622-627.

4 N. Lewinski, V. Colvin and R. Drezek, Small, 2008, 4, 26-49.

5 A. E. Nel, L. Madler, D. Velegol, T. Xia, E. M. V. Hoek, P. Somasundaran, F. Klaessig, V. Castranova and M. Thompson, Nat. Mater., 2009, 8, 543-557.

6 M. Deserno, Phys. Rev. E: Stat., Nonlinear, Soft Matter Phys., 2004, 69, 031903. 
7 H. J. Gao, W. D. Shi and L. B. Freund, Proc. Natl. Acad. Sci. U. S. A., 2005, 102, 9469-9474.

8 B. D. Chithrani, A. A. Ghazani and W. C. W. Chan, Nano Lett., 2006, 6, 662-668.

9 S. L. Zhang, J. Li, G. Lykotrafitis, G. Bao and S. Suresh, Adv. Mater., 2009, 21, 419-424.

10 T. T. Yue and X. R. Zhang, Soft Matter, 2011, 7, 9104-9112.

11 J. A. Champion and S. Mitragotri, Proc. Natl. Acad. Sci. U. S. A., 2006, 103, 4930-4934.

12 S. E. A. Gratton, P. A. Ropp, P. D. Pohlhaus, J. C. Luft, V. J. Madden, M. E. Napier and J. M. DeSimone, Proc. Natl. Acad. Sci. U. S. A., 2008, 105, 11613-11618.

13 K. Yang and Y. Q. Ma, Nat. Nanotechnol., 2010, 5, 579-583.

14 R. Vacha, F. J. Martinez-Veracoechea and D. Frenkel, Nano Lett., 2011, 11, 5391-5395.

15 R. Agarwal, V. Singh, P. Jurney, L. Shi, S. V. Sreenivasan and K. Roy, Proc. Natl. Acad. Sci. U. S. A., 2013, 110, 17247-17252.

16 S. Barua, J. W. Yoo, P. Kolhar, A. Wakankar, Y. R. Gokarn and S. Mitragotri, Proc. Natl. Acad. Sci. U. S. A., 2013, 110, 3270-3275.

17 S. Dasgupta, T. Auth and G. Gompper, Soft Matter, 2013, 9, 5473-5482.

18 Y. F. Li, H. Y. Yuan, A. von dem Bussche, M. Creighton, R. H. Hurt, A. B. Kane and H. J. Gao, Proc. Natl. Acad. Sci. U. S. A., 2013, 110, 12295-12300.

19 Y. Li, M. Kroger and W. K. Liu, Nanoscale, 2015, 7, 16631-16646.

20 Y. Li, T. T. Yue, K. Yang and X. R. Zhang, Biomaterials, 2012, 33, 4965-4973.

21 X. H. Shi, A. von dem Bussche, R. H. Hurt, A. B. Kane and H. J. Gao, Nat. Nanotechnol., 2011, 6, 714-719.

22 A. H. Bahrami, Soft Matter, 2013, 9, 8642-8646.

23 C. J. Huang, Y. Zhang, H. Y. Yuan, H. J. Gao and S. L. Zhang, Nano Lett., 2013, 13, 4546-4550.

24 K. Yang, B. Yuan and Y. Q. Ma, Nanoscale, 2013, 5, 7998-8006.

25 S. Dasgupta, T. Auth and G. Gompper, Nano Lett., 2014, 14, 687-693.

26 X. Yi, X. H. Shi and H. J. Gao, Nano Lett., 2014, 14, 1049-1055.

27 X. Yi, X. H. Shi and H. J. Gao, Phys. Rev. Lett., 2011, 107, 098101.

28 X. Yi and H. J. Gao, Phys. Rev. E: Stat., Nonlinear, Soft Matter Phys., 2014, 89, 062712.

29 Y. Li, X. R. Zhang and D. P. Cao, Nanoscale, 2015, 7, 2758-2769.

30 J. S. Sun, L. Zhang, J. L. Wang, Q. Feng, D. B. Liu, Q. F. Yin, D. Y. Xu, Y. J. Wei, B. Q. Ding, X. H. Shi and X. Y. Jiang, Adv. Mater., 2015, 27, 1402-1407.

31 L. Zhang, Q. Feng, J. L. Wang, S. Zhang, B. Q. Ding, Y. J. Wei, M. D. Dong, J. Y. Ryu, T. Y. Yoon, X. H. Shi, J. S. Sun and X. Y. Jiang, ACS Nano, 2015, 9, 9912-9921.

32 A. Verma, O. Uzun, Y. H. Hu, Y. Hu, H. S. Han, N. Watson, S. L. Chen, D. J. Irvine and F. Stellacci, Nat. Mater., 2008, 7, 588-595.

33 Y. F. Li, X. J. Li, Z. H. Li and H. J. Gao, Nanoscale, 2012, 4, 3768-3775.

34 Y. Li, M. Kroger and W. K. Liu, Biomaterials, 2014, 35, 8467-8478.
35 J. L. Wang, Y. J. Wei, X. H. Shi and H. J. Gao, RSC Adv., 2013, 3, 15776-15782.

36 B. D. Chithrani and W. C. W. Chan, Nano Lett., 2007, 7, 1542-1550.

37 K. Jaskiewicz, A. Larsen, I. Lieberwirth, K. Koynov, W. Meier, G. Fytas, A. Kroeger and K. Landfester, Angew. Chem., Int. Ed., 2012, 51, 4613-4617.

38 K. Jaskiewicz, A. Larsen, D. Schaeffel, K. Koynov, I. Lieberwirth, G. Fytas, K. Landfester and A. Kroeger, ACS Nano, 2012, 6, 7254-7262.

39 B. J. Reynwar, G. Illya, V. A. Harmandaris, M. M. Muller, K. Kremer and M. Deserno, Nature, 2007, 447, 461-464.

40 C. van der Wel, A. Vahid, A. Šarić, T. Idema, D. Heinrich and D. J. Kraft, Sci. Rep., 2016, 6, 32825.

41 I. Koltover, J. O. Radler and C. R. Safinya, Phys. Rev. Lett., 1999, 82, 1991-1994.

42 A. Saric and A. Cacciuto, Phys. Rev. Lett., 2012, 108, 118101. 43 A. H. Bahrami, R. Lipowsky and T. R. Weikl, Phys. Rev. Lett., 2012, 109, 188102.

44 A. Saric and A. Cacciuto, Phys. Rev. Lett., 2012, 109, 188101. 45 M. Raatz, R. Lipowsky and T. R. Weikl, Soft Matter, 2014, 10, 3570-3577.

46 M. Raatz and T. R. Weikl, Adv. Mater. Interfaces, 2017, 4, 1600325. 47 J. L. Wang, H. M. Yao and X. H. Shi, J. Mech. Phys. Solids, 2014, 73, 151-165.

48 H. Z. Zhang, Q. J. Ji, C. J. Huang, S. L. Zhang, B. Yuan, K. Yang and Y. Q. Ma, Sci. Rep., 2015, 5, 10525.

49 Y. Li, B. Yuan, K. Yang, X. R. Zhang, B. Yan and D. P. Cao, Nanotechnology, 2017, 28, 085102.

50 T. T. Yue and X. R. Zhang, ACS Nano, 2012, 6, 3196-3205.

51 L. P. Chen, S. Y. Xiao, H. Zhu, L. Wang and H. J. Liang, Soft Matter, 2016, 12, 2632-2641.

52 R. H. Guo, J. Mao and L. T. Yan, ACS Nano, 2013, 7, 10646-10653.

53 T. T. Yue and X. R. Zhang, Soft Matter, 2013, 9, 559-569.

54 H. M. Ding and Y. Q. Ma, Small, 2015, 11, 1055-1071.

55 S. L. Zhang, H. J. Gao and G. Bao, ACS Nano, 2015, 9, 8655-8671.

56 I. R. Cooke and M. Deserno, J. Chem. Phys., 2005, 123, 224710.

57 I. R. Cooke, K. Kremer and M. Deserno, Phys. Rev. E: Stat., Nonlinear, Soft Matter Phys., 2005, 72, 011506.

58 M. Simunovic and G. A. Voth, Nat. Commun., 2015, 6, 7219.

59 S. Plimpton, J. Comput. Phys., 1995, 117, 1-19.

60 J. C. Shillcock and R. Lipowsky, Nat. Mater., 2005, 4, 225-228.

61 A. Grafmuller, J. Shillcock and R. Lipowsky, Biophys. J., 2009, 96, 2658-2675.

62 R. M. Bhaskara, S. M. Linker, M. Vogele, J. Kofinger and G. Hummer, ACS Nano, 2017, 11, 1273-1280.

63 J. M. Hernandez, A. Stein, E. Behrmann, D. Riedel, A. Cypionka, Z. Farsi, P. J. Walla, S. Raunser and R. Jahn, Science, 2012, 336, 1581-1584.

64 A. H. Bahrami, M. Raatz, J. Agudo-Canalejo, R. Michel, E. M. Curtis, C. K. Hall, M. Gradzielski, R. Lipowsky and T. R. Weikl, Adv. Colloid Interface Sci., 2014, 208, 214-224. 